TAIWANESE JOURNAL OF MATHEMATICS

Vol. 14, No. 2, pp. 373-387, April 2010

This paper is available online at http://www.tjm.nsysu.edu.tw/

\title{
ON RANK 2 GEOMETRIES OF THE MATHIEU GROUP $M_{23}$
}

\author{
Nayil Kilic
}

\begin{abstract}
In this paper we determine all rank 2 geometries for the Mathieu group $M_{23}$ for which object stabilizers are maximal subgroups.
\end{abstract}

\section{INTRODUCTION}

In this paper, we give the list of all rank 2 geometries for $M_{23}$ that are firm, residually connected and flag transitive. We get 170 geometries of rank 2 . These results were checked using a series of MAGMA [1] programs. The paper is organized as follows. In section 2 , we recall the basic definitions needed in order to understand this paper. In section 3, we give the list of geometries we obtained. Finally, in section 4, we give the diagram of some rank 2 geometries for $M_{23}$.

\section{Definitions and Notation}

We begin by reviewing geometries and some standard notation. A geometry is a triple $(\Gamma, I, \star)$ where $\Gamma$ is a set, $I$ an index set and $\star$ a symmetric incidence relation on $\Gamma$ which satisfy

(i) $\Gamma=\cup_{i \in I} \Gamma_{i}$; and

(ii) if $x \in \Gamma_{i}, y \in \Gamma_{j}(i, j \in I)$ and $x \star y$, then $i \neq j$.

The elements of $\Gamma_{i}$ are called objects of type $i$, and $|I|$ is the rank of the geometry $\Gamma$ (as is usual we use $\Gamma$ is place of the triple $(\Gamma, I, \star)$ ). A flag $F$ of $\Gamma$ is a subset of $\Gamma$ in which every two elementof $F$ are incident. The rank of $F$ is $|F|$, the corank of $F$ is $|I \backslash F|$ and the type of $F$ is $\left\{i \in I \mid F \cap \Gamma_{i} \neq \emptyset\right\}$. A chamber of $\Gamma$ is a flag of type $I$. All geometries we consider are assumed to contain at least one flag of

Received February 20, 2008, accepted April 30, 2008.

Communicated by Ruibin Zhang.

2000 Mathematics Subject Classification: 20D08, 51E10, 05 C25.

Key words and phrases: Mathieu groups, Steiner system, Group geometries. 
rank $|I|$. The automorphism group of $\Gamma, A u t \Gamma$, consists of all permutations of $\Gamma$ which preserve the sets $\Gamma_{i}$ and the incidence relation $\star$. Let $G$ be a subgroup of $A u t \Gamma$. We call $\Gamma$ a flag transitive geometry for $G$ if for any two flags $F_{1}$ and $F_{2}$ of $\Gamma$ having the same type, there exists $g \in G$ such that $F_{1}{ }^{g}=F_{2}$. For $\Delta \subseteq \Gamma$, the residue of $\Delta$, denoted $\Gamma_{\Delta}$, is defined to be $\{x \in \Gamma \mid x \star y$ for all $y \in \Delta\}$. A geometry $\Gamma$ is called residually connected if for all flags $F$ of $\Gamma$ of corank 2 the incidence graph of $\Gamma_{F}$ is connected. We call $\Gamma$ firm provided that every flag of rank $|I|-1$ is contained in at least two chambers. The diagram of a firm, residually connected, flag-transitive geometry $\Gamma$ is a complete graph $K$, whose vertices are the elements of the set of type $I$ of $\Gamma$, provided with some additional structure which is further described as follows. To each vertex $i \in I$, we attach the order $s_{i}$ which is $\left|\Gamma_{F}\right|-1$ where $F$ is any flag of type $I \backslash\{i\}$, and the number $n_{i}$ of varieties of type $i$, which is the index of $G_{i}$ in $G$, and the subgroup $G_{i}$. To every edge $\{i, j\}$ of $K$, we associate three positive integers $d_{i j}, g_{i j}$ and $d_{j i}$ where $g_{i j}$ (the gonality) is equal to half the girth of the incidence graph of a residue $\Gamma_{F}$ of type $\{i, j\}$, and $d_{i j}$ (resp. $d_{j i}$ ), the $i$-diameter (resp. $j$-diameter) is the greatest distance from some fixed $i$ - element ( resp. $j$ - element ) to any other element in $\Gamma_{F}$.

On a picture of the diagram, this structure will often be depicted as follows.

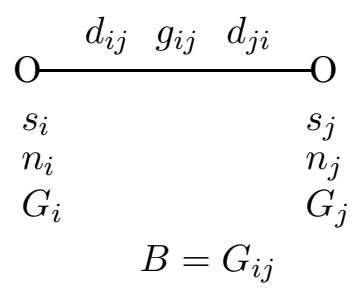

Now suppose that $\Gamma$ is a flag transitive geometry for the group $G$. As is wellknown we may view $\Gamma$ in terms of certain cosets of $G$. This is the approach we shall follow here. For each $i \in I$ choose an $x_{i} \in \Gamma_{i}$ and set $G_{i}=\operatorname{Stab}_{G}\left(x_{i}\right)$. Let $\mathcal{F}$ $=\left\{G_{i}: i \in I\right\}$. We now define a geometry $\Gamma(G, \mathcal{F})$ where the objects of type $i$ in $\Gamma(G, \mathcal{F})$ are the right cosets of $G_{i}$ in $G$ and for $G_{i} x$ and $G_{j} y(x, y \in G, i, j \in I)$ $G_{i} x \star G_{j} y$ whenever $G_{i} x \cap G_{j} y \neq \emptyset$. Also by letting $G$ act upon $\Gamma(G, \mathcal{F})$ by right multiplication we see that $\Gamma(G, \mathcal{F})$ is a flag transitive geometry for $G$. Moreover $\Gamma$ and $\Gamma(G, \mathcal{F})$ are isomorphic geometries for $G$. So we shall be studying geometries of the form $\Gamma(G, \mathcal{F})$, where $G \cong M_{23}$ and $G_{i}$ is a maximal subgroup of $G$ for all $i \in I$

Buekenhout, in [2], sought to give a wider view of geometries so as to encompass configurations observed in the finite sporadic simple groups. An outgrowth of this has been attemps to catalogue various subcollections of geometries for the finite sporadic simple groups (and other related groups). So - called minimal parabolic geometries and maximal 2-local geometries were investigated in [17] and [18] while geometries satisfying certain additional conditions for a number of (relatively) small 
order simple groups have been exhaustively examined. See, for example, $[3,6,11$, $12,13,16,14,15]$ and [19]. the results in most of these papers were obtained using various computer algebra systems. All rank 2 and rank 3 residually connected geometries of $M_{22}$ (the Mathieu Group of degree 22) were investigated in [9]. Again Kilic, in [10], calculated all rank 2 geometries for the Mathieu group $M_{24}$ for which object stabilizers are maximal subgroups. Now we determine all rank 2 geometries of $M_{23}$ (the Mathieu Group of degree 23) whose object stabilizer are maximal subgroups.

For the remainder of this paper $G$ will denote $M_{23}$, the Mathieu Group of degree 23. Also $\Omega$ will denote a 24 element set possessing the Steiner system $S(24,8,5)$ as described by Curtis's MOG [5]. We will follow the notation of [5].

So $\Omega=$\begin{tabular}{|l|l|l|}
$O_{1}$ & $O_{2}$ & $O_{3}$ \\
\hline
\end{tabular}$=$\begin{tabular}{ccc|cc|cc|}
$\infty$ & 14 & 17 & 11 & 22 & 19 \\
0 & 8 & 4 & 13 & 1 & 9 \\
3 & 20 & 16 & 7 & 12 & 5 \\
15 & 18 & 10 & 2 & 21 & 6 \\
\hline
\end{tabular} , where $O_{1}, O_{2}$ and $O_{3}$ are the heavy

bricks of the MOG. Here $M_{24}$ is the Mathieu group of degree 24 which leaves invariant the Steiner system $S(24,8,5)$ on $\Omega$. Set $\Lambda=\Omega \backslash\{24\}$

An octad of $\Omega$ is just an 8-element block of the Steiner system and a subset of $\Omega$ is called a dodecad if it is the symmetric difference of two octads of $\Omega$ which intersect in a set of size two. Corresponding to each 4 points of $\Omega$ there is a partition of the 24 points into 6 tetrads with the property that the union of any two tetrads is an octad, this configuration will be called a sextet. The following sets will appear when we describe geometries for $G$.

(i) $\mathcal{D}=\{X \subseteq \Lambda|| X \mid=2\}$ (duads of $\Lambda$ ).

(ii) $\mathcal{H}=\{X \subseteq \Lambda \mid X \cup\{\infty\}$ is an octad of $\Omega\}$ (heptads of $\Lambda$ ).

(iii) $\mathcal{O}=\{X \subseteq \Omega \mid X$ is an octad of $\Omega\}$ (octads of $\Lambda$ ).

(iv) $\mathcal{D}_{\mathrm{o}}=\{X \subseteq \Lambda \mid X$ is a dodecad of $\Omega\}$ (dodecads of $\Lambda$ ).

(v) $\mathcal{S}=\left\{X_{i} \subseteq \Omega|| X_{i} \mid=4\right.$ (for each $\left.i \in I\right), X_{i} \cup X_{j}$ is an octad $(i \neq j$ ) and $\left.\Omega=\cup_{i \in I} X_{i}, i \in I=\{1 \ldots 6\}\right\}$ (sextets of $\Omega$ ).

From the [4], the conjugacy classes of the maximal subgroups of $G$ are as follows:

\begin{tabular}{|l|l|l|l|}
\hline Order & Index & $M_{i}$ & Description \\
\hline 443520 & 23 & $M_{1} \cong M_{22}$ & $M_{1}=\operatorname{Stab}_{G}\{a\}, a \in \Lambda$ \\
40320 & 253 & $M_{2} \cong L_{3}(4): 2 b$ & $M_{2}=\operatorname{Stab}_{G}\{X\}, X \in \mathcal{D}$ \\
40320 & 253 & $M_{3} \cong 2^{4}: A_{7}$ & $M_{3}=\operatorname{Stab}_{G}\{X\}, X \in \mathcal{H}$ \\
20160 & 506 & $M_{4} \cong A_{8}$ & $M_{4}=\operatorname{Stab}_{G}\{X\}, X \in \mathcal{O}$ \\
7920 & 1288 & $M_{5} \cong M_{11}$ & $M_{5}=\operatorname{Stab}_{G}\{X\}, X \in \mathcal{D}_{\mathrm{o}}$ \\
5760 & 1771 & $M_{6} \cong 2^{4}:\left(3 \times A_{5}\right): 2$ & $M_{6}=\operatorname{Stab}_{G}\{X\}, X \in \mathcal{S}$ \\
253 & 40320 & $M_{7} \cong 23: 11$ & \\
\hline
\end{tabular}


For $i \in\{1, \ldots, 7\}$, we let $\mathfrak{M}_{\mathrm{i}}$ denote the conjugacy class of $M_{i}, M_{i}$ as given in the previous table. We also set $\mathfrak{M}=\bigcup_{i=1}^{7} \mathfrak{M}_{\mathfrak{i}}$; so $\mathfrak{M}$ consist of all maximal subgroups of $G$. In [5] and [10], we can find further information about 23:11. Also put $\mathfrak{X}=\Lambda \cup \mathcal{D} \cup \mathcal{H} \cup \mathcal{O} \cup \mathcal{D}_{\mathrm{o}} \cup \mathcal{S}$.

Suppose $G_{1}$ and $G_{2}$ are maximal subgroups of $G$ with $G_{1} \neq G_{2}$. Set $G_{12}=$ $G_{1} \cap G_{2}$. We use $\mathfrak{M}_{\mathrm{ij}}(t)$ to describe $\left\{G_{1}, G_{2}, G_{1} \cap G_{2}\right\}$ according to the following scheme: $G_{1} \in \mathfrak{M}_{\mathrm{i}}, G_{2} \in \mathfrak{M}_{\mathrm{j}}$ (and so $G_{1}=\operatorname{Stab}_{G}\left(X_{1}\right)$ and $G_{2}=\operatorname{Stab}_{G}\left(X_{2}\right)$ for some appropriate subsets $X_{1}$ and $X_{2}$ of $\Omega$ in $\mathfrak{X}$ ) with $\left|X_{1} \cap X_{2}\right|=t$. When listing up the rank 2 geometries of $G$ in Theorem 1 the notation $\mathfrak{M}_{\mathrm{ij}}(t)$ is not sufficient enough to describe the geometries up to conjugacy in AutG. All calculations in $2^{4}:\left(3 \times A_{5}\right): 2$ and $23: 11$ we can not use this notation, we shall use the following notation; $\mathfrak{M}_{46}(1)$ means the first case of the intersection of octad and sextet, $\mathfrak{M}_{46}(2)$ means the second case of the intersection of octad and sextet, using the same kind of idea we can define the remaining geometries. In addition, up to conjugacy, there are two geometries, we denote them the same notation as in $\mathfrak{M}_{47}(1)$ (where $G_{12}=1$ for both geometries). Similarly, up to conjugacy, we find 5 geometries for $\mathfrak{M}_{57}(1)$, 2 geometries for $\mathfrak{M}_{66}(1), 87$ geometries for $\mathfrak{M}_{77}(1), 7$ geometries for $\mathfrak{M}_{67}(1), 2$ geometries for $\mathfrak{M}_{77}(2)$.

Two geometries $\Gamma_{1}\left(G,\left\{G_{1}, G_{2}\right\}\right)$ and $\Gamma_{2}\left(G,\left\{H_{1}, H_{2}\right\}\right)$ are conjugate iff there exists an element $g \in G$ such that $\left\{G_{1}, G_{2}\right\}^{g}=\left\{H_{1}, H_{2}\right\}$. In this paper, N.G. denotes the number of geometries up to conjugacy.

Below we give certain subsets of $\Lambda$ which will be encountered frequently in our

list. $H_{1}=$
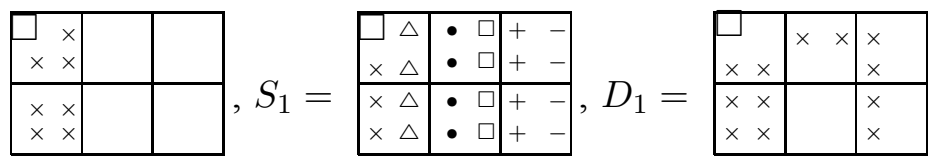

$O_{2}=$\begin{tabular}{|l|l|l|}
\hline & $\bullet$ & \\
\hline & $\because$ & \\
& $\bullet$ &
\end{tabular} \mid , where $H_{1} \in \mathcal{H}, S_{1} \in \mathcal{S}, D_{1} \in \mathcal{D}_{\mathrm{o}}$ and $O_{2} \in \mathcal{O}$.

Our notation is as in the [4] with the following addition: $F_{n}$ a Frobenious group of order $\mathrm{n}$ and $\left(S_{n} \times S_{m}\right)^{+}$is the group of even permutation in the permutation group $S_{n} \times S_{m}$.

\section{RANK 2 GeOMEtries of $M_{23}$}

In this section, we give, up to conjugacy, all rank 2 geometries for $M_{23}$ that are firm, residually connected and flag transitive. 
Theorem 1. Up to conjugacy in AutG there are 170 firm, residually connected and flag transitive rank 2 geometries of $\Gamma=\Gamma\left(G,\left\{G_{1}, G_{2}\right\}\right)$ with $G_{1}, G_{2} \in \mathfrak{M}$. These together with the shape and order of $G_{12}$ are listed in the following Table.

\begin{tabular}{|c|c|c|c|c|c|c|c|}
\hline $\bar{\Gamma}$ & $G_{12}$ & $G_{12}$ & $N . G$. & $\Gamma$ & $G_{12}$ & $\left|G_{12}\right|$ & $N . G$ \\
\hline $\mathfrak{M}_{11}(0)$ & $M_{21}$ & 20160 & 1 & $\mathfrak{M}_{12}(1)$ & $L_{3}(4)$ & 20160 & 1 \\
\hline $\mathfrak{M}_{12}(0)$ & $2^{4} \cdot S_{5}$ & 1920 & 1 & $\mathfrak{M}_{13}(1)$ & $2^{4} \cdot A_{6}$ & 5760 & 1 \\
\hline $\mathfrak{M}_{13}(0)$ & $A_{7}$ & 2520 & 1 & $\mathfrak{M}_{14}(1)$ & $A_{7}$ & 2520 & 1 \\
\hline$\overline{\mathfrak{M}_{14}(0)}$ & $2^{3} \cdot L_{3}(2)$ & 1344 & 1 & $\mathfrak{M}_{15}(0)$ & $L_{2}(11)$ & 660 & 1 \\
\hline $\mathfrak{M}_{15}(1)$ & $M_{10}$ & 720 & 1 & $\mathfrak{M}_{16}(1)$ & $2^{4} .3^{2} .2$ & 288 & 1 \\
\hline $\mathfrak{M}_{16}(2)$ & $2^{4} . S_{5}$ & 1920 & 1 & $\mathfrak{M}_{17}(1)$ & $C_{11}$ & 11 & 1 \\
\hline$\overline{\mathfrak{M}_{22}(0)}$ & $\overline{2}^{4} \cdot D_{12}$ & $\overline{192}$ & $\overline{11}$ & $\overline{\mathfrak{M}_{22}(1)}$ & $\overline{2^{4} \cdot S L(2,4)}$ & $\overline{960}$ & $\overline{11}$ \\
\hline $\mathfrak{M}_{23}(0)$ & $2 \times L_{3}(2)$ & 336 & 1 & $\mathfrak{M}_{23}(1)$ & $A_{6}$ & 360 & 1 \\
\hline $\mathfrak{M}_{23}(2)$ & $2^{4} . S_{5}$ & 1920 & 1 & $\mathfrak{M}_{24}(0)$ & $2^{4} \cdot D_{12}$ & 192 & 1 \\
\hline $\mathfrak{M}_{24}(2)$ & $\overline{S_{6}}$ & 720 & 1 & $\mathfrak{M}_{24}(1)$ & $L_{3}(2)$ & 168 & 1 \\
\hline $\mathfrak{M}_{25}(0)$ & $M_{9.2}$ & 144 & 1 & $\mathfrak{M}_{25}(2)$ & $\overline{S_{5}}$ & 120 & 1 \\
\hline $\mathfrak{M}_{25}(1)$ & $A_{5}$ & 60 & 1 & $\mathfrak{M}_{26}(1)$ & $2^{4} \cdot D_{12}$ & 192 & 1 \\
\hline $\mathfrak{M}_{26}(2)$ & $3^{2} \cdot 2^{2}$ & 36 & 1 & $\mathfrak{M}_{26}(3)$ & $2^{4} . S_{5}$ & 1920 & 1 \\
\hline $\mathfrak{M}_{26}(4)$ & $2^{4} . S_{3}$ & 96 & 1 & $\mathfrak{M}_{27}(1)$ & 1 & 1 & 1 \\
\hline$\overline{\mathfrak{M}_{33}(3)}$ & 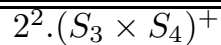 & 2288 & 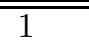 & $\overline{\overline{\mathfrak{M}_{33}(1)}}$ & 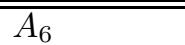 & 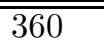 & 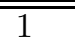 \\
\hline $\mathfrak{M}_{34}(4)$ & $2^{2} \cdot\left(S_{3} \times S_{4}\right)^{+}$ & 288 & 1 & $\mathfrak{M}_{34}(2)$ & $S_{5}$ & 120 & 1 \\
\hline $\mathfrak{M}_{34}(0)$ & $2^{3} \cdot L_{3}(2)$ & 1344 & 1 & $\mathfrak{M}_{35}(6)$ & $A_{6}$ & 360 & 1 \\
\hline $\mathfrak{M}_{35}(4)$ & $M_{8} . S_{3}$ & 48 & 1 & $\mathfrak{M}_{35}(2)$ & $S_{5}$ & 120 & 1 \\
\hline $\mathfrak{M}_{36}(1)$ & $2^{4} .\left(S_{4} \times S_{3}\right)^{+}$ & 1152 & 1 & $\mathfrak{M}_{36}(2)$ & $2 \times S_{4}$ & 48 & 1 \\
\hline $\mathfrak{M}_{36}(3)$ & $\left(S_{4} \times S_{3}\right)^{+}$ & 72 & 1 & $\mathfrak{M}_{36}(4)$ & $S_{5}$ & 120 & 1 \\
\hline $\mathfrak{M}_{37}(1)$ & 1 & 1 & 1 & & & & \\
\hline $\mathfrak{M}_{44}(0)$ & $2^{3} \cdot L_{3}(2)$ & 1344 & 1 & $\mathfrak{M}_{44}(4)$ & $2^{4} . S_{3}$ & 96 & 1 \\
\hline $\mathfrak{M}_{44}(2)$ & $\left(S_{4} \times S_{3}\right)^{+}$ & 72 & 1 & $\mathfrak{M}_{45}(2)$ & $S_{5}$ & 120 & 1 \\
\hline $\mathfrak{M}_{45}(4)$ & $\overline{S_{4}}$ & 24 & 1 & $\mathfrak{M}_{45}(6)$ & $3^{2} \cdot D_{8}$ & 72 & 1 \\
\hline $\mathfrak{M}_{46}(1)$ & $2^{4} .3^{2} \cdot 2^{2}$ & 576 & 1 & $\mathfrak{M}_{46}(2)$ & $2^{2} \cdot D_{12}$ & 48 & 1 \\
\hline $\mathfrak{M}_{46}(3)$ & $S_{4}$ & 24 & 1 & $\mathfrak{M}_{46}(4)$ & $\left(S_{3} \times S_{5}\right)^{+}$ & 360 & 1 \\
\hline $\mathfrak{M}_{46}(5)$ & $2 \times S_{4}$ & 48 & 1 & $\mathfrak{M}_{47}(1)$ & 1 & 1 & 2 \\
\hline $\mathfrak{M}_{55}(8)$ & $S_{4}$ & 24 & 1 & $\mathfrak{M}_{55}(6)$ & $D_{10}$ & 10 & 1 \\
\hline $\mathfrak{M}_{55}(4)$ & $M_{8} . S_{3}$ & 48 & 1 & $\mathfrak{M}_{56}(1)$ & $3^{2} .2^{2}$ & 36 & 1 \\
\hline $\mathfrak{M}_{56}(2)$ & $D_{12}$ & 12 & 1 & $\mathfrak{M}_{56}(3)$ & $F_{20}$ & 20 & 1 \\
\hline $\mathfrak{M}_{56}(4)$ & $S_{4}$ & 24 & 1 & $\mathfrak{M}_{56}(5)$ & $M_{8} \cdot S_{3}$ & 48 & 1 \\
\hline $\mathfrak{M}_{57}(1)$ & $C_{11}$ & 11 & 5 & $\mathfrak{M}_{57}(2)$ & 1 & 1 & 1 \\
\hline $\mathfrak{M}_{66}(1)$ & $D_{12}$ & 12 & 2 & $\mathfrak{M}_{66}(2)$ & $3^{2} .2^{2}$ & 36 & 1 \\
\hline $\mathfrak{M}_{66}(3)$ & $2^{4} .2^{2}$ & 64 & 1 & $\mathfrak{M}_{66}(4)$ & $2^{4} . S_{3}$ & 96 & 1 \\
\hline $\mathfrak{M}_{66}(5)$ & $2^{4} .3^{2} .2$ & 288 & 1 & $\mathfrak{M}_{67}(1)$ & 1 & 1 & 7 \\
\hline $\mathfrak{M}_{77}(1)$ & 1 & 1 & 87 & $\mathfrak{M}_{77}(2)$ & $C_{11}$ & 11 & 2 \\
\hline
\end{tabular}

We now define some subgroups for $\mathfrak{M}_{\mathrm{ij}}(\mathrm{x})(x \in\{1,2 \ldots, 5\}, i \in\{1,2 \ldots, 7\}, j=$ $\{6\})$ by giving the $X_{l} \in \mathfrak{X}$ such that $G_{l}=\operatorname{Stab}_{G} X_{l}(l=1,2)$. 


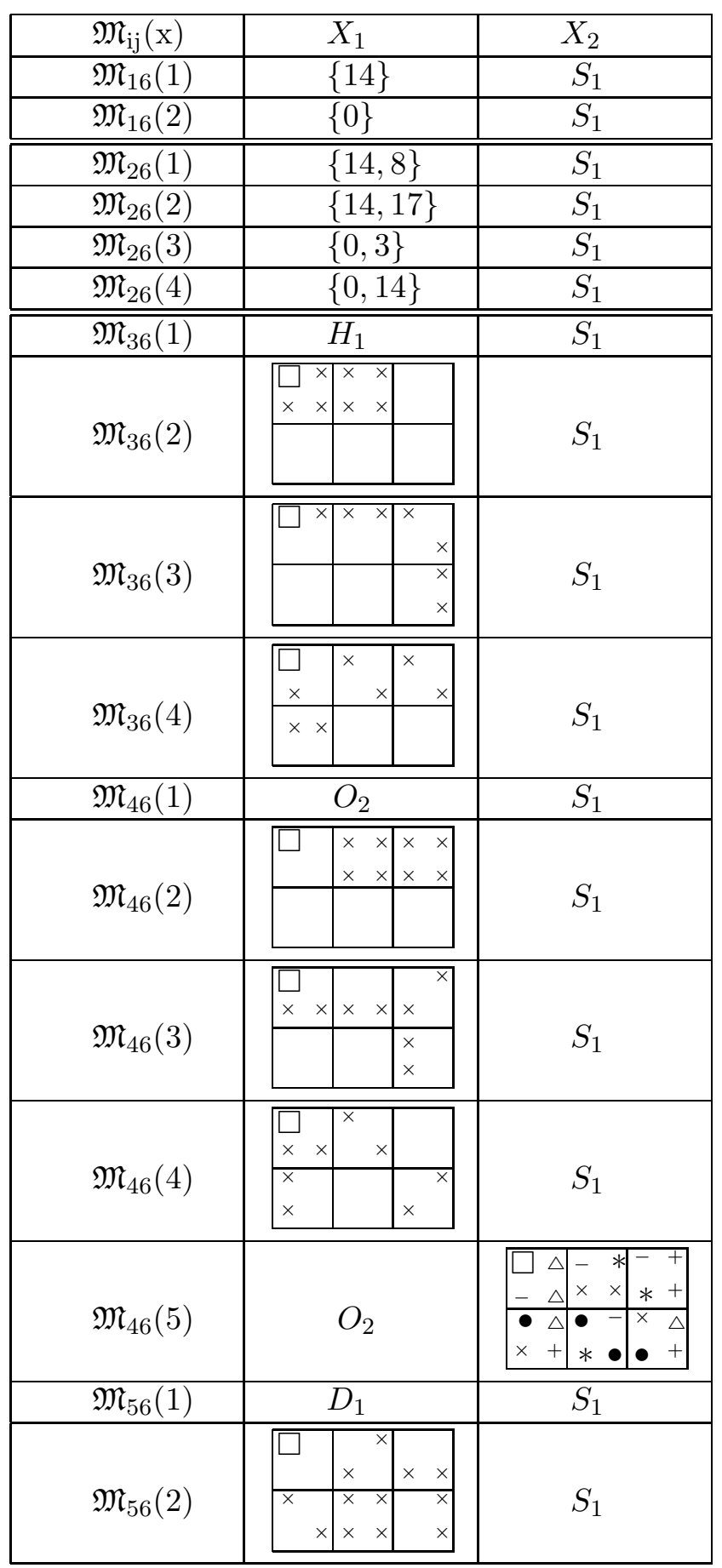




\begin{tabular}{|c|c|c|c|c|}
\hline $\mathfrak{M}_{56}(3)$ & 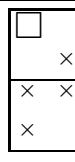 & \begin{tabular}{|r|r|} 
& \\
$\times$ & \\
$\times$ & $\times$ \\
& $\times$ \\
\end{tabular} & $\begin{array}{|ll|}x & \\
x & x \\
& x \\
\end{array}$ & $S_{1}$ \\
\hline $\mathfrak{M}_{56}(4)$ & \begin{tabular}{|ll} 
& \\
$\times$ & $\times$ \\
$\times$ & $\times$ \\
\end{tabular} & 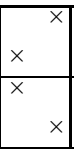 & \begin{tabular}{|l}
$x$ \\
$x$ \\
$x$ \\
$x$ \\
\end{tabular} & $S_{1}$ \\
\hline $\mathfrak{M}_{56}(5)$ & & $\begin{array}{r} \\
\times \quad \times \\
\times \quad \times \\
\times \\
\times \quad \times \\
\end{array}$ & \begin{tabular}{|l|l|}
$\times$ & \\
$\times$ & $x$ \\
& $\times$ \\
\end{tabular} & $S_{1}$ \\
\hline $\mathfrak{M}_{66}(1)$ & & $S_{1}$ & & 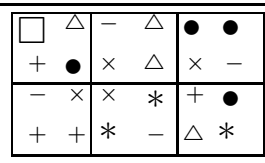 \\
\hline $\mathfrak{M}_{66}(2)$ & & $S_{1}$ & & \begin{tabular}{|cc|cc|cc|} 
& $\bullet$ & + & - & $\times$ & $\bullet$ \\
$\times$ & $\triangle$ & $\times$ & $\times$ & $*$ & $\Delta$ \\
- & $\bullet$ & $*$ & + & + & $\Delta$ \\
+ & $\bullet$ & - & $*$ & - & $\Delta$ \\
\end{tabular} \\
\hline $\mathfrak{M}_{66}(3)$ & & $S_{1}$ & & 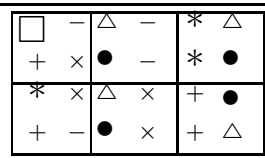 \\
\hline $\mathfrak{M}_{66}(4)$ & & $S_{1}$ & & \begin{tabular}{|cc|cc|cc|} 
& $\Delta$ & - & - & $\Delta$ & + \\
$*$ & $\bullet$ & $\bullet$ & $\Delta$ & $\bullet$ & + \\
+ & - & $\times$ & $\bullet$ & - & $*$ \\
$*$ & $\times$ & $\Delta$ & $\times$ & $\times$ & + \\
\end{tabular} \\
\hline $\mathfrak{M}_{66}(5)$ & & $S_{1}$ & & 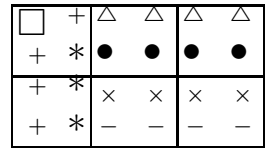 \\
\hline
\end{tabular}

\section{Diagrams for Rank 2 Geometries}

In this section, we give the diagrams of the following geometries.

\begin{tabular}{|l|c|l|c|l|c|}
\hline$\Gamma$ & Diagrams & $\Gamma$ & Diagrams & $\Gamma$ & Diagrams \\
\hline $\mathfrak{M}_{11}(0)$ & 4.1 & $\mathfrak{M}_{12}(1)$ & 4.2 & $\mathfrak{M}_{12}(0)$ & 4.3 \\
\hline $\mathfrak{M}_{13}(0)$ & 4.4 & $\mathfrak{M}_{13}(1)$ & 4.5 & $\mathfrak{M}_{14}(0)$ & 4.6 \\
\hline $\mathfrak{M}_{14}(1)$ & 4.7 & $\mathfrak{M}_{15}(1)$ & 4.8 & $\mathfrak{M}_{15}(0)$ & 4.9 \\
\hline $\mathfrak{M}_{16}(2)$ & 4.10 & $\mathfrak{M}_{16}(1)$ & 4.11 & $\mathfrak{M}_{22}(0)$ & 4.12 \\
\hline $\mathfrak{M}_{22}(1)$ & 4.13 & $\mathfrak{M}_{23}(0)$ & 4.14 & $\mathfrak{M}_{23}(1)$ & 4.15 \\
\hline $\mathfrak{M}_{23}(2)$ & 4.16 & $\mathfrak{M}_{24}(0)$ & 4.17 & $\mathfrak{M}_{24}(2)$ & 4.18 \\
\hline
\end{tabular}

(Continued) 


\begin{tabular}{|l|c|l|c|l|c|}
\hline$\Gamma$ & Diagrams & $\Gamma$ & Diagrams & $\Gamma$ & Diagrams \\
\hline $\mathfrak{M}_{24}(1)$ & 4.19 & $\mathfrak{M}_{25}(0)$ & 4.20 & $\mathfrak{M}_{25}(2)$ & 4.21 \\
\hline $\mathfrak{M}_{25}(1)$ & 4.22 & $\mathfrak{M}_{26}(1)$ & 4.23 & $\mathfrak{M}_{26}(2)$ & 4.24 \\
\hline $\mathfrak{M}_{26}(3)$ & 4.25 & $\mathfrak{M}_{26}(4)$ & 4.26 & $\mathfrak{M}_{33}(3)$ & 4.27 \\
\hline $\mathfrak{M}_{33}(1)$ & 4.28 & $\mathfrak{M}_{34}(4)$ & 4.29 & $\mathfrak{M}_{34}(2)$ & 4.30 \\
\hline $\mathfrak{M}_{34}(0)$ & 4.31 & $\mathfrak{M}_{35}(6)$ & 4.32 & $\mathfrak{M}_{35}(4)$ & 4.33 \\
\hline $\mathfrak{M}_{35}(2)$ & 4.34 & $\mathfrak{M}_{36}(1)$ & 4.35 & $\mathfrak{M}_{36}(2)$ & 4.36 \\
\hline $\mathfrak{M}_{36}(3)$ & 4.37 & $\mathfrak{M}_{36}(4)$ & 4.38 & $\mathfrak{M}_{44}(0)$ & 4.39 \\
\hline $\mathfrak{M}_{44}(4)$ & 4.40 & $\mathfrak{M}_{44}(2)$ & 4.41 & $\mathfrak{M}_{45}(2)$ & 4.42 \\
\hline $\mathfrak{M}_{45}(4)$ & 4.43 & $\mathfrak{M}_{45}(6)$ & 4.44 & $\mathfrak{M}_{46}(1)$ & 4.45 \\
\hline $\mathfrak{M}_{46}(2)$ & 4.46 & $\mathfrak{M}_{46}(3)$ & 4.47 & $\mathfrak{M}_{46}(4)$ & 4.48 \\
\hline $\mathfrak{M}_{46}(5)$ & 4.49 & $\mathfrak{M}_{55}(8)$ & 4.50 & $\mathfrak{M}_{55}(6)$ & 4.51 \\
\hline $\mathfrak{M}_{55}(4)$ & 4.52 & $\mathfrak{M}_{56}(1)$ & 4.53 & $\mathfrak{M}_{56}(2)$ & 4.54 \\
\hline $\mathfrak{M}_{56}(3)$ & 4.55 & $\mathfrak{M}_{56}(4)$ & 4.56 & $\mathfrak{M}_{56}(5)$ & 4.57 \\
\hline $\mathfrak{M}_{66}(3)$ & 4.58 & $\mathfrak{M}_{66}(1)$ & 4.59 & $\mathfrak{M}_{66}(2)$ & 4.60 \\
\hline $\mathfrak{M}_{66}(4)$ & 4.61 & $\mathfrak{M}_{66}(5)$ & 4.62 & $\mathfrak{M}_{77}(1)$ & 4.63 \\
\hline $\mathfrak{M}_{77}(2)$ & 4.64 & $\mathfrak{M}_{77}(1)$ & 4.65 & $\mathfrak{M}_{57}(2)$ & 4.66 \\
\hline $\mathfrak{M}_{67}(1)$ & 4.67 & $\mathfrak{M}_{57}(1)$ & 4.68 & $\mathfrak{M}_{47}(1)$ & 4.69 \\
\hline
\end{tabular}

In above table, $\mathfrak{M}_{77}(1)$ and $\mathfrak{M}_{77}(1)^{*}$ denote different geometries.

$$
\begin{aligned}
& 4.1 \mathrm{O}-3 \quad 2 \quad 3-\mathrm{O} \\
& \begin{array}{lc}
21 & 21 \\
23 & 23 \\
M_{22} & M_{22}
\end{array}
\end{aligned}
$$

Due to Leemans (see [16], geometry 2.1).

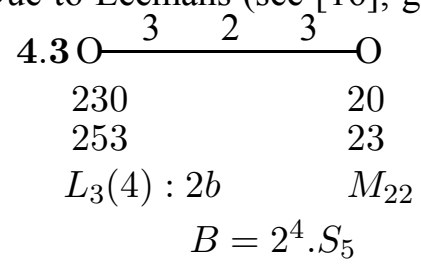

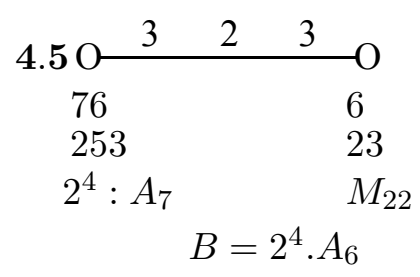

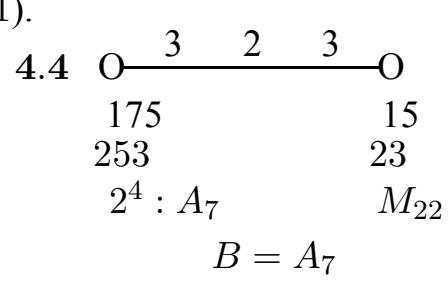

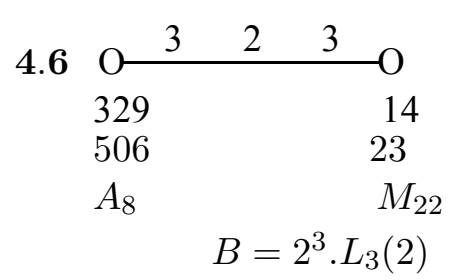



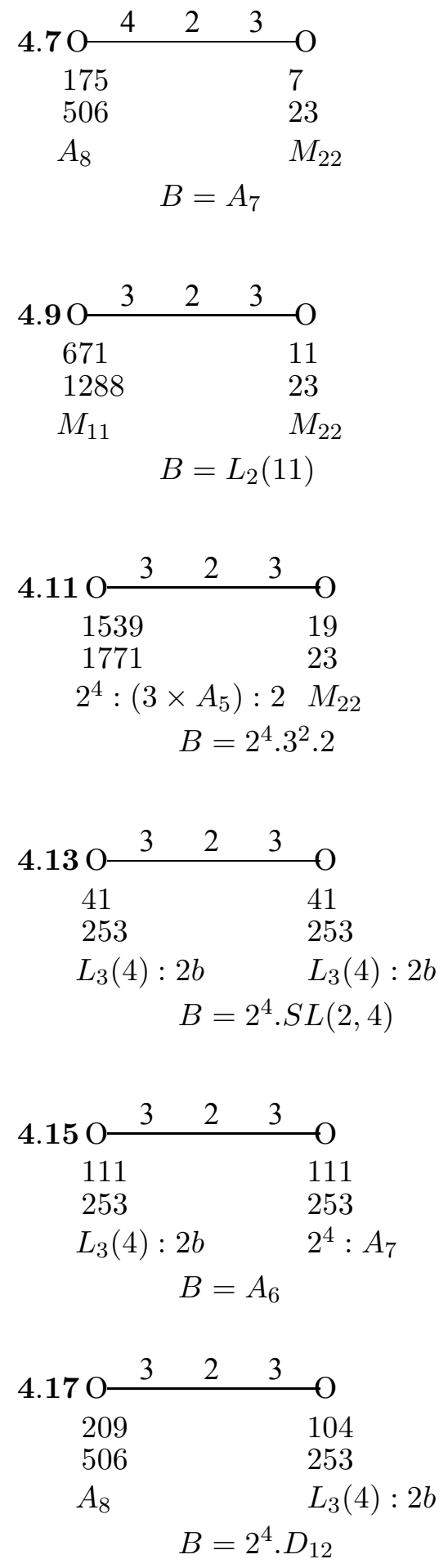
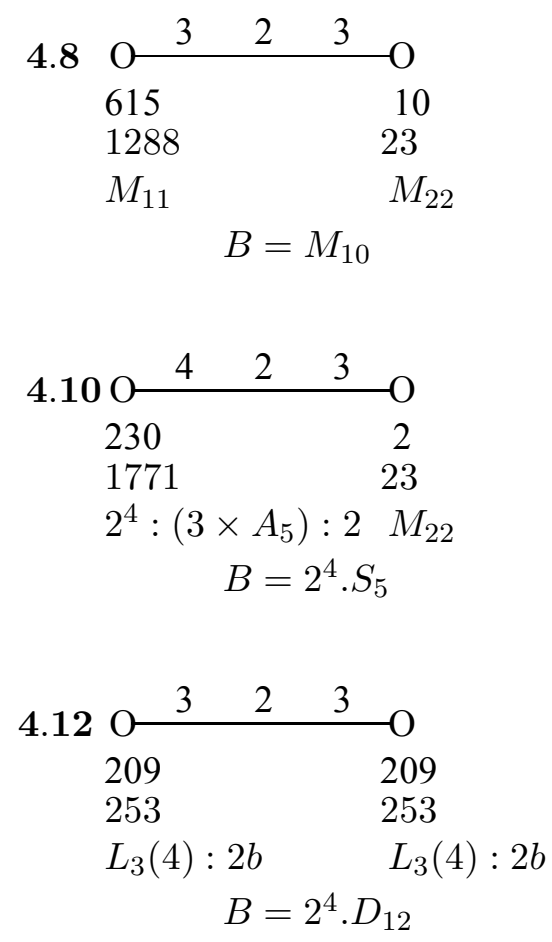
$4.14 \mathrm{O}-3 \quad 2 \quad 3 \quad \mathrm{O}$

$119 \quad 119$

$253 \quad 253$
$2^{4}: A_{7} \quad L_{3}(4): 2 b$ $B=2 \times L_{3}(2)$

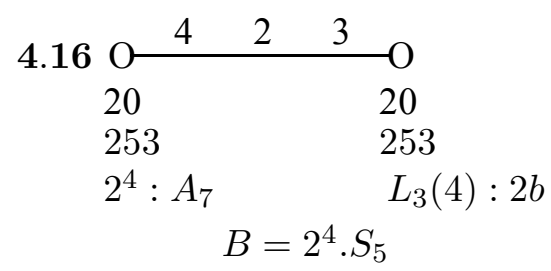
$4.18 \mathrm{O} \quad 4 \quad 2 \quad 3 \quad \mathrm{O}$
$55 \quad 27$
$506 \quad 253$
$A_{8} \quad L_{3}(4): 2 b$ $B=S_{6}$



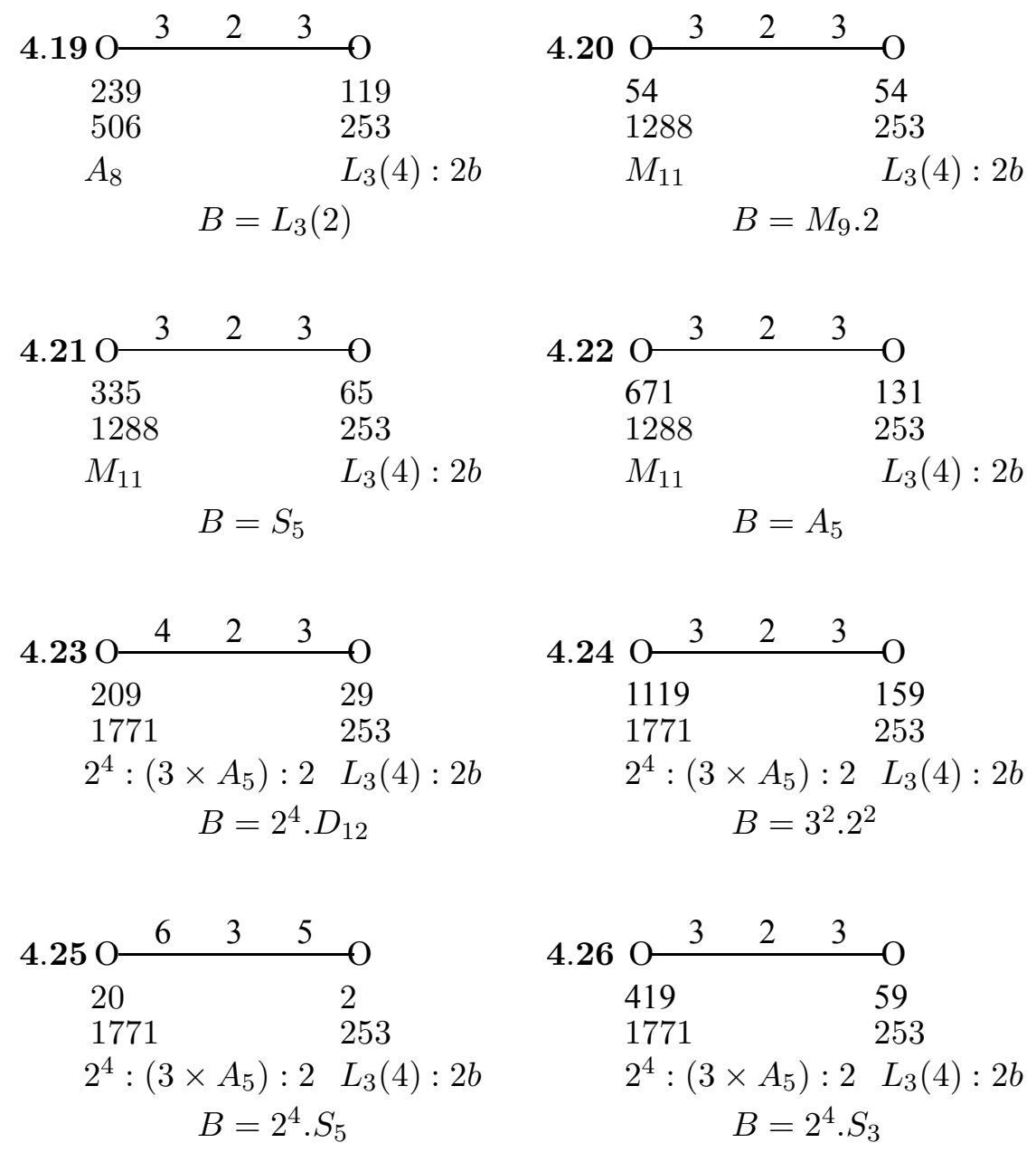

Due to Leemans (see [16], geometry 2.2).
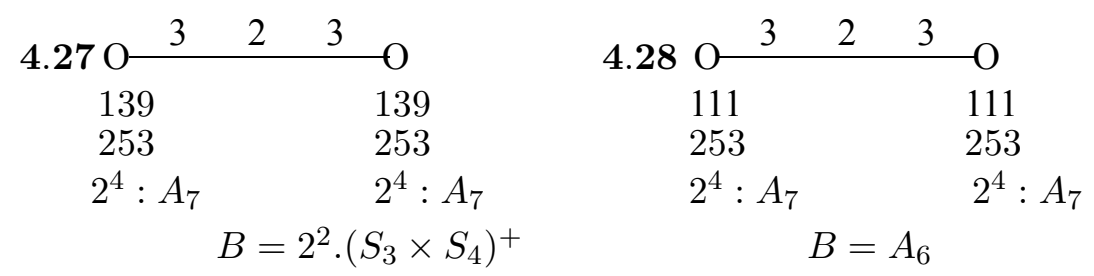


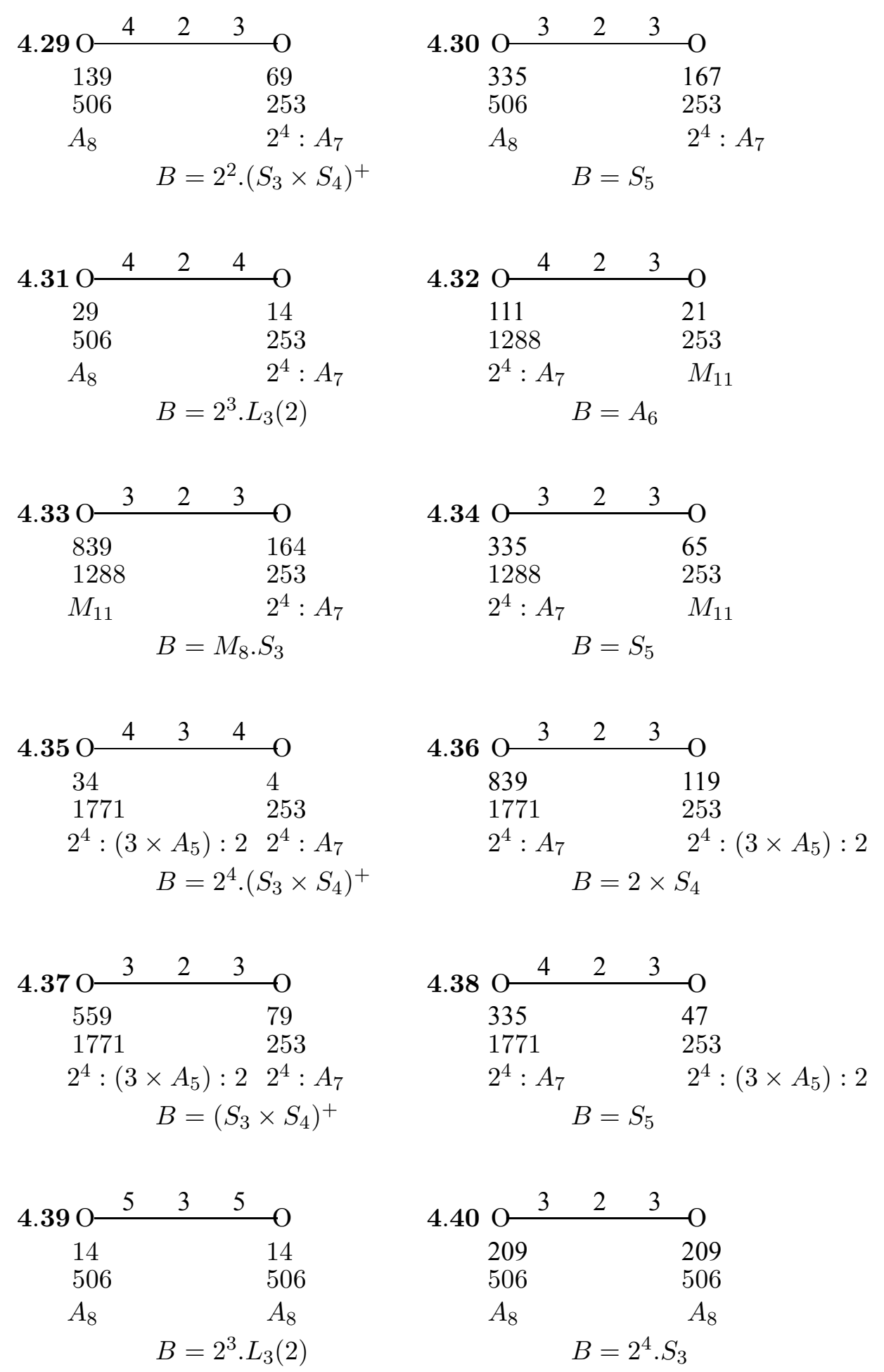


Due to Leemans (see [16], geometry 2.4).

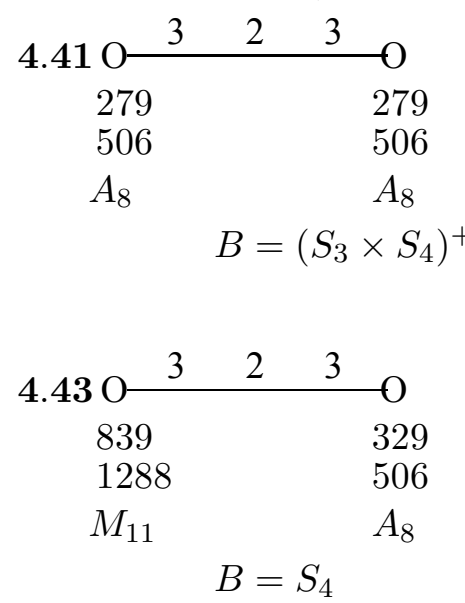

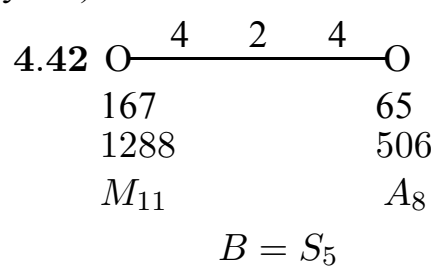

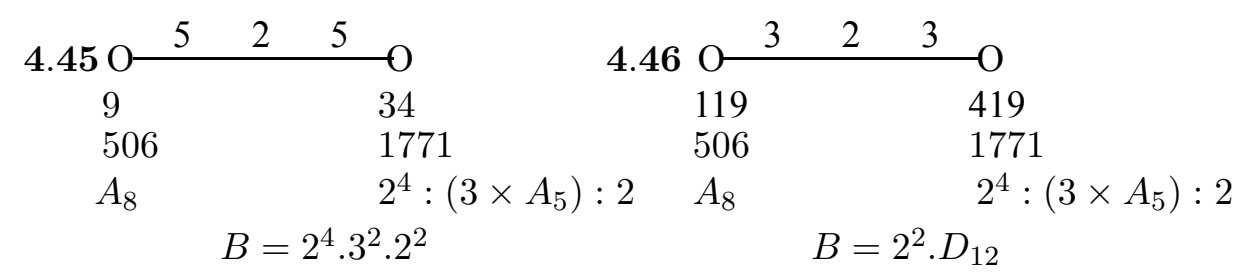

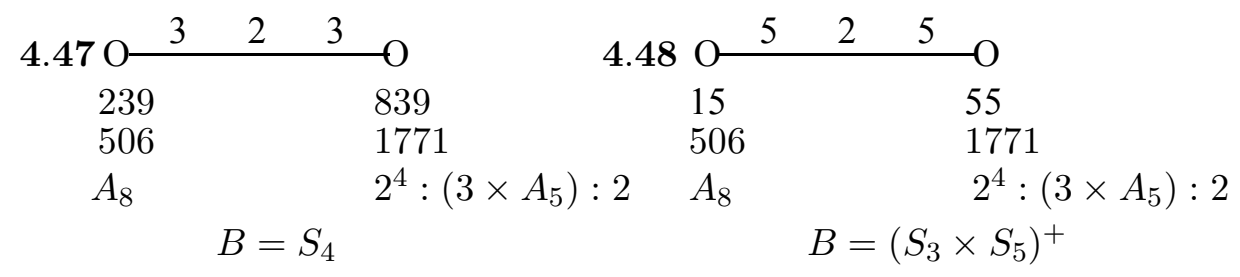

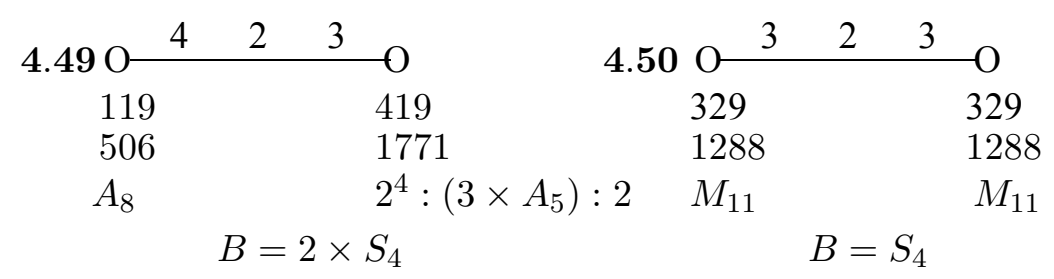



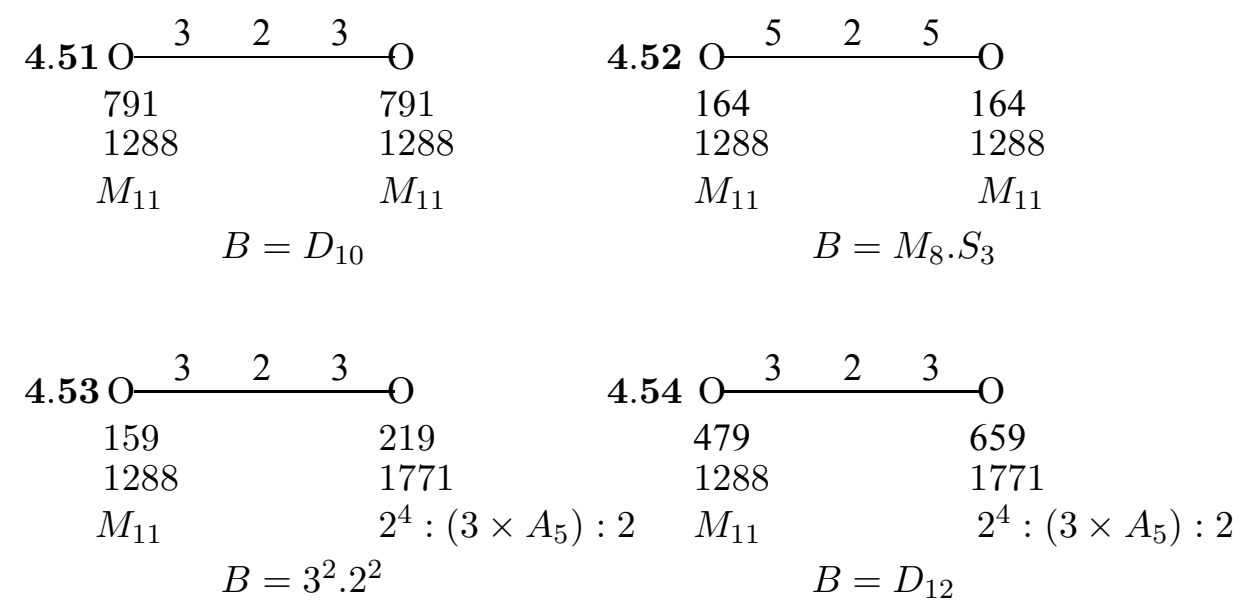

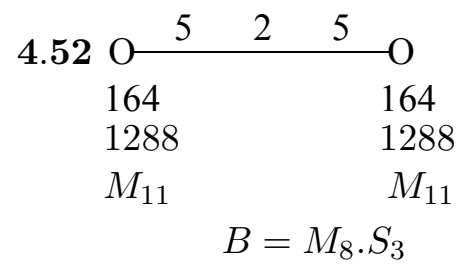

$B=D_{12}$

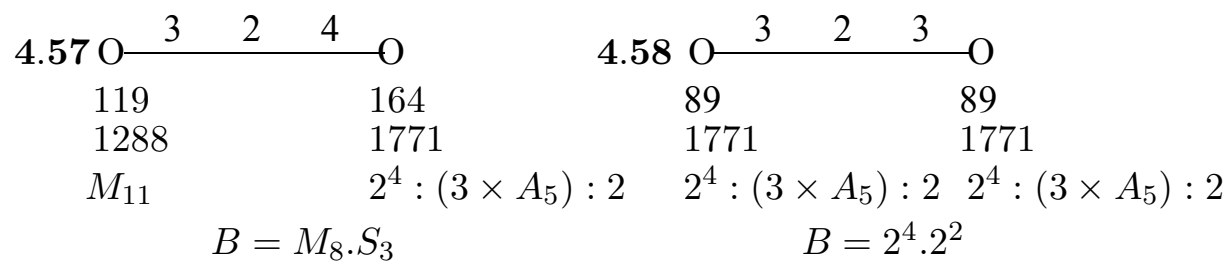

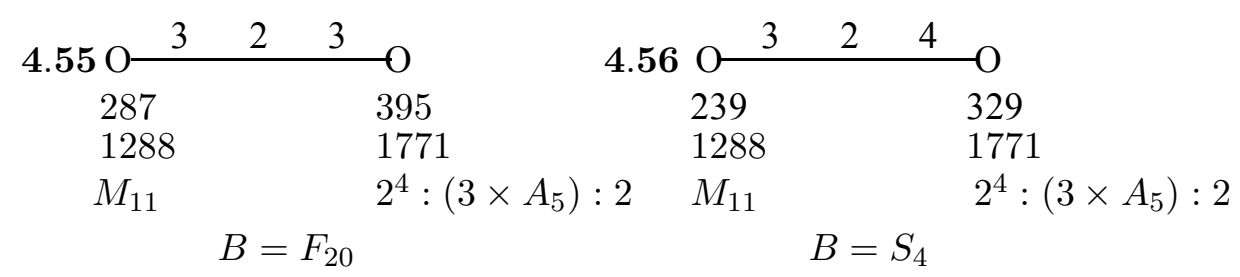

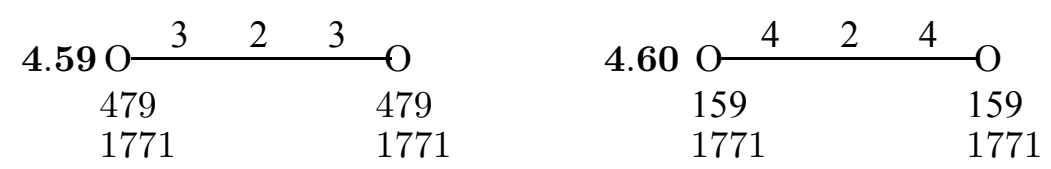

$2^{4}:\left(3 \times A_{5}\right): 2 \quad 2^{4}:\left(3 \times A_{5}\right): 2 \quad 2^{4}:\left(3 \times A_{5}\right): 2 \quad 2^{4}:\left(3 \times A_{5}\right): 2$

$$
B=D_{12} \quad B=3^{2} .2^{2}
$$

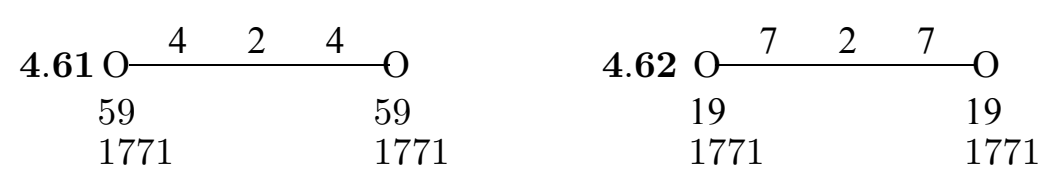

$$
\begin{array}{cc}
2^{4}:\left(3 \times A_{5}\right): 22^{4}:\left(3 \times A_{5}\right): 2 & 2^{4}:\left(3 \times A_{5}\right): 2 \quad 2^{4}:\left(3 \times A_{5}\right): 2 \\
B=2^{4} . S_{3} & B=2^{4} .3^{2} .2
\end{array}
$$




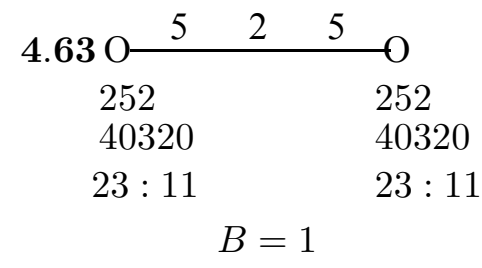

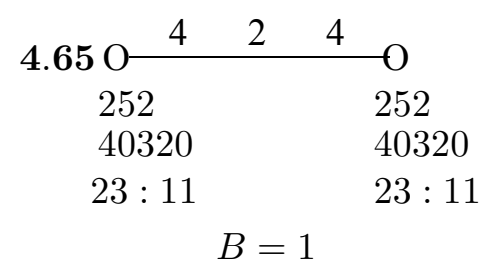

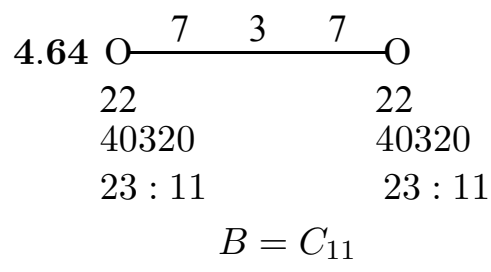

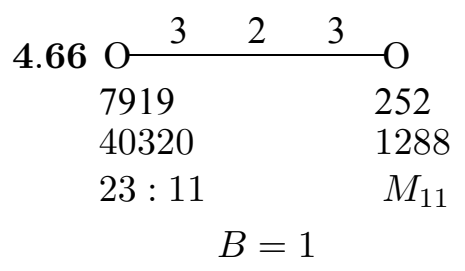

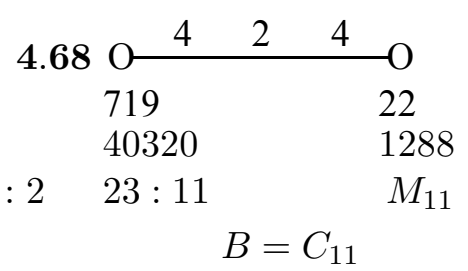

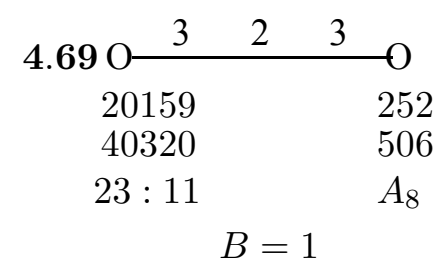

\section{REFERENCES}

1. W. Bosma, J. Cannon and C. Playoust, The Magma Algebra System I: the user language, J. Symbolic Comput., 3/4 (1997), 235-265.

2. F. Buekenhout, The basic diagram of a geometry, Lecture Notes, Springer, Vol. 893, 1981.

3. F. Buekenhout, M. Dehon and D. Leemans, All geometries of the Mathieu group $M_{11}$ based on maximal subgroups, Experimental Math., 5 (1996), 101-110.

4. J. H. Conway, R. T. Curtis, S. P. Norton, R. A. Parker and R. A. Wilson, An Atlas of Finite Groups, Oxford Univ. Press, London, 1985.

5. R. T. Curtis, A new combinatorial approach to $M_{24}$, Math. Proc. Camb. Phil. Soc., 79 (1976), 25-42. 
6. M. Dehon and D. Leemans, Constructing coset geometries with Magma: An application to the sporadic groups $M_{12}$ and $J_{1}$, Atti Sem. Mat. Fis. Univ. Modena, L (2002), 415-427.

7. The GAP Group, GAP-Groups, Algorithms, and Programming, Version 4.4:2005, (http://www.gap-system.org).

8. N. Kilic, Residually connected geometries for $M_{22}$, Ph.D. thesis, UMIST, Manchester, 2002.

9. N. Kilic and P. Rowley, On rank 2 and rank 3 residually connected geometries for $M_{22}$, Note di Matematica, 22 (2003), 107-154.

10. N. Kilic, On rank 2 geometries of the Mathieu group $M_{24}$, Preprint, 2005.

11. E. A. Komissartschik and S. V. Tsaranov, Construction of finite groups amalgams and geometries. Geometries of the group $U_{4}(2)$, Commun. Algebra, 18(N4) (1990), 1071-1117.

12. D. Leemans, The rank 3 geometries of the simple Suzuki group $S z(q)$. Note Mat., 19 (1999), 43-64.

13. D. Leemans, The residually weakly primitive pre-geometry of the Suzuki simple groups, Note Mat., 20 (2001), 1-20.

14. D. Leemans, The residually weakly primitive geometries of $J_{2}$, Note Mat., 21 (2002). nr. $1,77-81$.

15. D. Leemans, The residually weakly primitive geometries of $M_{22}$. Designs, Codes, Crypto., 29 (2003). n.r. 1/2/3, 177-178.

16. D. Leemans, The residually weakly primitive geometries of $M_{23}$, Atti Sem. Mat. Fis. Univ. Modena e Reggio Emilia, LII (2004), 313-316.

17. M. A. Ronan and S. D. Smith, 2-local geometries for some sporadic groups, AMS Symposia in Pure Mathematics 37 (Finite Groups). American Math. Soc., 1980, pp. 283-289.

18. M. A. Ronan and G. Stroth, Minimal parabolic geometries for the sporadic groups, Europ. J. Combinatorics, 5 (1984), 59-91.

19. S. V. Tsaranov, Geometries and amalgams of $J_{1}$, Comm. Algebra, 18(N4) (1990), 1119-1135.

\author{
Nayil Kilic \\ Department of Mathematics, \\ Arts and Science Faculty, \\ Harran University, \\ Sanliurfa 63000, \\ Turkey \\ E-mail: nayilkilic@harran.edu.tr
}

